\title{
Exploring social inequalities in healthcare trajectories following diagnosis of diabetes: a state sequence analysis of linked survey and administrative data
}

Rachel McKay ${ }^{1,2}$, Laurence Letarte ${ }^{3,4}$, Alexandre Lebel ${ }^{3,4}$, Amélie Quesnel-Vallée ${ }^{1,2,5^{*}}$ and on behalf of the TORSADE Cohort Working Group

\begin{abstract}
Background: Social inequalities in complications associated with diabetes mellitus persist. As a primary care sensitive condition (PCSC), this association could be related to differential access to primary care. Our objectives are to establish a typology of care trajectories following a new diagnosis, and to explore social determinants of trajectories.

Methods: We used the TorSaDe (The Care Trajectories-Enriched Data) cohort, which links Canadian Community Health Survey respondents to health administrative data. Care trajectories were mapped over a two-year period following a new diagnosis and analysed using state sequence and clustering methods. Associations between individual and geographic characteristics with trajectory types were assessed with multinomial logistic regression.

Results: Three trajectories were identified: Regular Family Physician (FP) Predominant, Specialist Physician Predominant, and Few Services. With Regular FP as the reference, males had higher odds of experiencing the Few Services trajectory, higher education was associated with higher odds of both the Few Services and the Specialist trajectories, and immigrants had higher odds of the Specialist trajectory. Diagnoses in a physician's office, as opposed to in hospital, were associated with higher odds of the Regular FP trajectory.

Conclusions: The Regular FP trajectory most closely aligns with the management principles of the PCSC approach. We did not find strong evidence of social status privileging access to this trajectory. However, the association with location of diagnosis suggests that efforts to ensure patients diagnosed in hospital are well linked to a regular family physician for follow up may help to reduce unnecessary specialist use and meet PCSC goals.
\end{abstract}

\section{Introduction}

The global prevalence of diabetes has been on the rise for the past several decades [1]. The potential over time for damage to the heart, blood vessels, eyes, kidneys, and nerves necessitates long-term clinical follow up and care [1]. The care trajectory concept describes the sequence of healthcare use over time [2]. Understanding the pattern of care set in motion by the diagnosis of diabetes will support the identification of care trajectories that minimize unnecessary or inappropriate services while maximizing health outcomes, and as such has important implications for health system design and for improving patient experiences.

\footnotetext{
${ }^{*}$ Correspondence: Amelie.quesnelvallee@mcgill.ca

${ }^{1}$ McGill Observatory on Health and Social Services Reforms, Montreal,

Canada

Full list of author information is available at the end of the article
} 


\section{Primary care for quality diabetes care}

Good control of diabetes (maintaining an average HbA1c below $6.5 \%, 48 \mathrm{mmol} / \mathrm{mol}$ ) in the first year following a diabetes diagnosis has been associated with reduced risk of complications and death 10 years later, even after adjusting for glycemic control after the first year [3]. This "legacy effect" of early control highlights the importance of ensuring patients are appropriately connected to support and service resources following diagnosis. Considered a primary care sensitive condition (PCSC) [4], effective management is highly contingent on timely access to quality primary care, and notably continuity of care $(\mathrm{CoC})$, which is characterised by an ongoing, cooperative relationship between a patient and their physician-led care team [5]. Higher $\mathrm{CoC}$ has been associated with lower risk of preventable hospitalization following a new diagnosis of diabetes [6].

\section{Socioeconomic disparities in care}

Lower socioeconomic status, as well as sociodemographics including education level, sex, and immigrant-status, have been linked to poorer diabetes outcomes - notably through health behaviours, but also poorer access to health care (even in settings with universal insurance) and quality of care [7-10]. These studies often use summary indicators of healthcare access, which can limit information about the order and timing of care in the trajectory. An understanding of these elements is relevant to improving interventions in diabetes management, considering the "legacy effect" of early control of diabetes. Longitudinal studies of the patterns of healthcare use could play a critical role in our understanding of how inequalities in diabetes care arise - and hence, where interventions could limit their progression into inequalities in avoidable morbidity.

With this in mind, we aimed to explore care trajectories defined by states of care as ascribed to interactions across the health care system, in order to better represent the order, timing, and continuity of care. Our main objectives were to assess patterns of care trajectories in the 2 years following a diabetes diagnosis, and to explore social determinants of these patterns.

\section{Methods}

\section{Study design}

This was a retrospective cohort study in the province of Quebec, Canada. The study was approved by the Commission d'accès à l'information, l'Institut de statistique du Québec, and McGill's Research Ethics Board. All methods were performed in accordance with the relevant guidelines and regulations. The need for informed consent was waived by McGill's Research Ethics Board as this was a retrospective analysis of de-identified administrative data.

\section{Data sources}

We used the Care Trajectories - Enriched Data (Tor$\mathrm{SaDE})$ cohort for this study, which is a linkage of Canadian Community Health Survey (CCHS) and health administrative data. Phase 1 of the cohort consists of a sample of 61,083 consenting Quebec CCHS respondents (from survey years 2007 to 2012) and 19years of health administrative data [11].

Briefly, the CCHS is a comprehensive population-based cross-sectional health survey with self-reported information on health status and health determinants. As our goal was to make inference about the TorSaDE cohort, and not the Canadian population, we opted not to conduct weighted analyses. This decision was in alignment with other studies using linked survey and administrative data in similar ways $[12,13]$.

As elsewhere in Canada, Quebec has a universal health insurance program, covering residents of the province for all medically necessary physician and hospital services. Health administrative data correspond to all physician visits reimbursed on a fee-for-service basis, and all hospital visits (1996 to 2016). Data include the date, diagnosis code, patient and physician identifiers, and service descriptors.

To identify a subcohort of individuals with incident diabetes, we used a validated algorithm of two physician visits or one hospitalization with a diabetes code in a 2 year period [14]. To ensure our subcohort represented people with incident cases, we restricted the subsample to cases without any previous diabetes code registered in the 5 years preceding the incident date, and excluded females with pregnancy-related events within 5 months of the diagnosis [15]. Our subcohort included adults aged 20 years and older, to eliminate the period of transition from pediatric to adult programs that occurs at age 18 [16].

\section{Care trajectories}

The index date was the day of diabetes diagnosis, as identified by the algorithm [14]. We followed the care trajectory for 2 years, beginning on the day after diagnosis.

To create the care trajectories, we identified all physician and hospital encounters in the follow-up period for each individual. We included any visit diagnosis code, not solely diabetes-related visits, as we were interested in total healthcare use. Our care trajectories were organized by five states which were defined based on our review of the data and interpretation of important distinctions in care: regular family physician care, new family physician 
care, specialist care, emergency department or hospital care, and no health service. We defined a regular family physician (FP) as one with whom $\geq 50 \%$ of the individual's FP visits in the preceding 365 days had occurred. A new FP was defined as any with whom $<50 \%$ of these visits had occurred. An individual who had seen a physician on an outpatient basis was considered to be under the care of that physician for 90 days or until the next health care encounter to align with recommendations for follow up every 3 months for less well-controlled diabetes [17]. If no further health care encounter occurred before the expiration of the 90-day period, the individual was considered to not be under active care of a provider ("no health service"). In the present analysis, emergency department visits were grouped with hospitalizations due to relatively small numbers in these categories and the conceptual similarity in institution-based care states.

The trajectories initially lasted 730 days, with one state coded for each day based on the conditions described above. However, given the precondition that a diagnosis occurs in office or hospital, i.e., in an active state of care, the state of no health service only began to appear in the trajectories at day 90, causing a clear shift in the distribution of care states. We thus opted to begin our analysis of trajectories from day 91 to eliminate this issue. Sensitivity analyses showed the substantive results were unchanged. As such, the length of trajectories for this analysis is 641 days.

\section{Variables}

Age was recorded in the CCHS and recalculated at the start of the trajectory period. For social demographic measures, we used education level as a proxy for socioeconomic status, because this was a time-invariant measure. Education was categorized as 'No high school diploma, 'High school diploma,' 'Post-secondary diploma', 'University degree.' We also explored immigration status, recalculating the time since immigration to the start of the trajectory.

A Combined Charlson and Elixhauser Comorbidity index [18] using Schneeweiss weights [19] was calculated for the year prior to the start of the care trajectory, to adjust for individual-level baseline comorbidity differences. The index is the result of the sum of weights derived from 30-day mortality predictions for each identified condition [18], with a higher index indicating a higher morbidity. Place of diagnosis was based on whether the incidence algorithm flagged a case through physician visit (considered to be in office) or hospitalization (considered to be in hospital).

Geography was explored with a four-category zone variable (census metropolitan area (CMA) of Montreal; other CMA in the province; census agglomeration (CA); rural areas) that distinguished the largest urban centre of Montreal from other urban centres, smaller towns, and rural areas.

\section{Analysis}

The analysis was conducted in three steps. First, state sequence analysis was used to analyze the trajectories [20]. This approach takes every pair of trajectory sequences and applies a sequence alignment algorithm to derive a matrix of dissimilarity. We used the dynamic hamming distance (DHD) alignment algorithm (which does not use an indel cost) [21], with the substitution matrix based on time-varying state transitions. We opted to use the DHD algorithm as optimal matching was too computationally demanding; additionally, this approach was theoretically sound as DHD privileges the order and timing of events, in contrast to optimal matching which prioritizes the occurrence of states [22]. In our application, we assumed that differences in order and timing of the states would be meaningful.

In the second step, cluster analysis was performed on the dissimilarity matrix to group similar trajectories using an agglomerative hierarchical clustering procedure and Ward linkage method. The number of clusters was selected by visually inspecting the inertia curve and the clustering dendrogram and then assessing the meaningfulness of the resulting groups (see supplementary Figs. 1 and 2). After determining the number of clusters that best represented our data, we described the distribution of individuals assigned to each cluster.

As our final step, we assessed the relevance of covariates in predicting membership in each trajectory group using multivariable multinomial logistic regression models, given the polytomous outcome.

\section{Results \\ Descriptive}

Our subcohort consisted of 4308 individuals with an incident diagnosis of diabetes, $49 \%$ of which $(n=2112)$ were female (Table 1). The mean age was 62 years, $38 \%$ did not have a high school diploma, and $8 \%$ were immigrants. Three-quarters were diagnosed in a physician office setting, and $29 \%$ lived in Montreal, while one-third (33\%) lived in a rural location (Table 1). There was very little attrition during the study period, but the records of 130 individuals were removed due to death during follow-up. These deaths predictably occurred among older people (mean age 74 years, standard deviation 10.2), with higher pre-trajectory comorbidities (mean Combined Comorbidity Index score of 6.7, standard deviation 5.2, compared to a mean score of 0.9 , standard deviation 2.2, overall for the remaining cohort). 
Table 1 Distribution of patient characteristics by trajectory group

\begin{tabular}{|c|c|c|c|c|c|}
\hline Characteristic & & $\begin{array}{l}\text { Total sample } \\
(N=4308)\end{array}$ & $\begin{array}{l}\text { Regular GP } \\
(n=1854 ; 43 \%)\end{array}$ & $\begin{array}{l}\text { Specialist } \\
\text { predominant } \\
(n=1746 ; \\
41 \%)\end{array}$ & $\begin{array}{l}\text { Few services } \\
(n=708 ; 16 \%)\end{array}$ \\
\hline Age & Mean (SD) & $61.6(12.5)$ & $62.1(11.9)$ & $62.5(12.7)$ & $58.1(12.9)$ \\
\hline \multirow[t]{5}{*}{ Age groups } & $20-40$ & $240(6 \%)$ & $75(4 \%)$ & $105(6 \%)$ & $60(8 \%)$ \\
\hline & $41-60$ & $16,778(39 \%)$ & $743(40 \%)$ & $591(34 \%)$ & $344(49 \%)$ \\
\hline & $61-70$ & $1325(31 \%)$ & $558(30 \%)$ & $574(33 \%)$ & $193(27 \%)$ \\
\hline & $71-80$ & $811(19 \%)$ & $372(20 \%)$ & $365(21 \%)$ & $74(10 \%)$ \\
\hline & $80+$ & $254(6 \%)$ & $106(6 \%)$ & $111(6 \%)$ & $37(5 \%)$ \\
\hline $\begin{array}{l}\text { Combined Comorbidity Index in the year } \\
\text { before diagnosis }\end{array}$ & Mean (SD) & $0.87(2.2)$ & $0.6(1.9)$ & $1.23(2.5)$ & $0.60(2.1)$ \\
\hline \multirow[t]{5}{*}{ Education } & Some high school education & $1627(38 \%)$ & 779 (42\%) & $625(36 \%)$ & $223(31 \%)$ \\
\hline & High school diploma & $588(14 \%)$ & $249(13 \%)$ & $244(14 \%)$ & $95(13 \%)$ \\
\hline & College/CEGEP & $1572(36 \%)$ & $644(35 \%)$ & $636(36 \%)$ & $292(41 \%)$ \\
\hline & University degree & $481(11 \%)$ & $163(9 \%)$ & $227(13 \%)$ & $91(13 \%)$ \\
\hline & Missing & $40(1 \%)$ & $19(1 \%)$ & $14(1 \%)$ & $7(1 \%)$ \\
\hline \multirow[t]{2}{*}{ Sex } & Female & $2112(49 \%)$ & $921(50 \%)$ & $903(52 \%)$ & $288(41 \%)$ \\
\hline & Male & $2186(51 \%)$ & $923(50 \%)$ & $843(48 \%)$ & $420(59 \%)$ \\
\hline \multirow[t]{3}{*}{ Immigrant } & yes & $328(8 \%)$ & $104(6 \%)$ & $174(10 \%)$ & $50(7 \%)$ \\
\hline & no & $3977(92 \%)$ & $1748(94 \%)$ & $1571(90 \%)$ & $658(93 \%)$ \\
\hline & missing & & $2(0 \%)$ & $1(0 \%)$ & $0(0 \%)$ \\
\hline Years since immigration & Mean (SD) & $34.1(17.7)$ & $35.6(16.6)$ & $34.0(18.3)$ & $30.2(18.7)$ \\
\hline \multirow[t]{5}{*}{ Service use during trajectory Mean (SD) } & Regular GP & $8.9(7.5)$ & $11.9(8.8)$ & $7.7(5.6)$ & $3.7(2.8)$ \\
\hline & Specialist visits & $11.5(14.9)$ & $7.0(6.7)$ & $19.1(19.8)$ & $4.4(4.3)$ \\
\hline & Hospital days & $20.6(34.8)$ & $18.9(34.0)$ & $23.5(38.8)$ & $17.8(24.0)$ \\
\hline & Emergency visits & $4.2(5.2)$ & $3.6(3.7)$ & $5.2(6.8)$ & $3.4(3.4)$ \\
\hline & New GP visits & $4.2(4.7)$ & $3.5(3.5)$ & $5.2(5.9)$ & $3.4(3.3)$ \\
\hline \multirow[t]{2}{*}{ Place of diagnosis } & office & $3259(76 \%)$ & $1528(82 \%)$ & $1185(68 \%)$ & $546(77 \%)$ \\
\hline & hospital & $1049(24 \%)$ & $326(18 \%)$ & $561(32 \%)$ & $162(23 \%)$ \\
\hline \multirow[t]{5}{*}{ Residential Area (at start of trajectory) } & CMA of Montreal ${ }^{\mathrm{a}}$ & $1269(29 \%)$ & $460(25 \%)$ & $614(35 \%)$ & $195(28 \%)$ \\
\hline & Non-Montreal CMA & $868(20 \%)$ & $404(22 \%)$ & $339(19 \%)$ & $125(18 \%)$ \\
\hline & Census agglomeration (CA) & $731(17 \%)$ & $335(18 \%)$ & $265(15 \%)$ & $131(19 \%)$ \\
\hline & Rural area & $1420(33 \%)$ & $647(35 \%)$ & $526(30 \%)$ & $247(35 \%)$ \\
\hline & missing & $20(0 \%)$ & $8(0 \%)$ & $2(0 \%)$ & $10(1 \%)$ \\
\hline
\end{tabular}

${ }^{\text {a } C M A ~ C e n s u s ~ M e t r o p o l i t a n ~ A r e a ~}$

The sequence analysis and clustering procedure resulted in three distinct groups (see supplementary Figs. 1 and 2 for the dendrogram and inertia curves, respectively, which we named based on the predominant care states (Fig. 1).

The largest cluster (43\% of the sample) formed the pattern we call Regular FP Trajectory. Individuals following this trajectory type spent an average of 341 days (out of the 641, i.e., $53 \%$ of the trajectory days) in the regular family physician care state, and a small proportion of time in the hospital and emergency care state (mean $=26$ days, i.e., $4 \%$ of trajectory days) (Fig. 2).
The next largest group (41\%) followed a pattern we termed Specialist Trajectory. Individuals in this group spent an average of 330 days (51\%) in the specialist care state. They spent the most time, on average, of the three groups in the hospital and emergency care state (mean $=46$ days; $7 \%$ ).

Finally, $16 \%$ of the sample followed a pattern we call Few Services Trajectory, spending an average of 418 days (65\% of trajectory days) in a state with no services. This group had the lowest specialist and regular family physician involvement. 

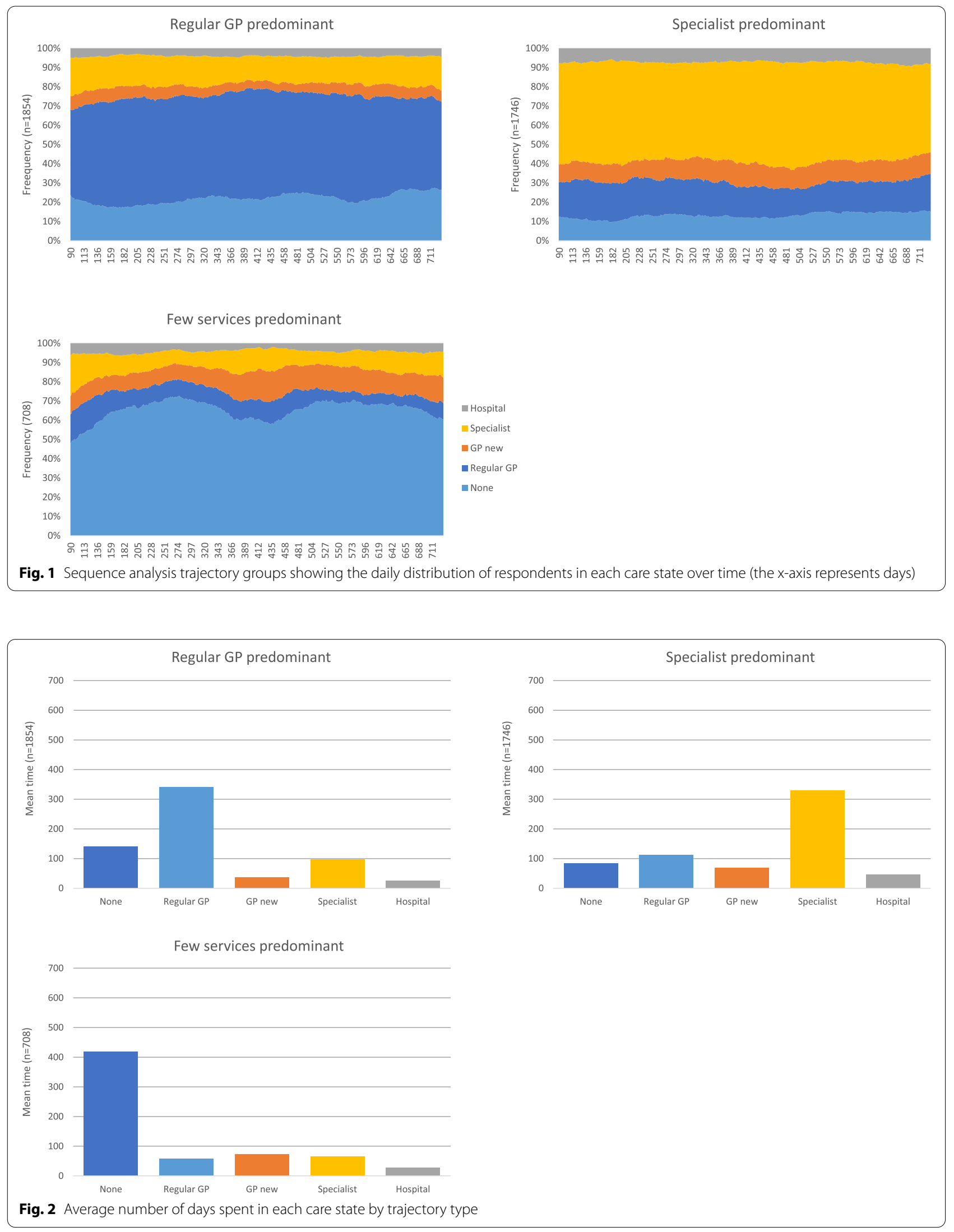
Respondents following the Specialist Trajectory spent a similar amount of time as the Few Services Trajectory members in the new family physician care state (mean $=69$ and 72 days, respectively), but nearly twice as much time as those members in the regular family physician care state (112 days vs. 58 days). People in the Regular FP Trajectory spent about the same amount of time in the hospital and emergency care state as the Few Services Trajectory members (mean $=26$ days and 27 days respectively) (Fig. 2).

While the distribution of men and women following the Regular FP Trajectory was evenly split, there was a higher proportion of men in the Few Services Trajectory, and a higher proportion of women in the Specialist Trajectory (Table 1). The Few Services and Specialist Trajectories had a higher proportion of people with a university degree. There was a higher proportion of immigrants in the Specialist than in the other trajectory groups. There were more younger people and fewer older people in the Few Services Trajectory. People living in the urban centre of Montreal at diagnosis had higher representation in the Specialist Trajectory.

\section{Model results}

Specialist vs. family physician

All of the model results presented are adjusted for all other covariates. Higher comorbidity was associated with higher odds of membership in the Specialist as compared to the Regular FP Trajectory (adjusted odds ratio, aOR 1.08, 95\% CI 1.05-1.12) (Fig. 3; see also supplementary Table 1). Diabetes diagnosis in a physician's office, as compared to in hospital, was associated with lower odds of membership in the Specialist Trajectory (aOR 0.47, 95\% CI 0.39-0.56). Higher levels of education were associated with higher odds of membership in this trajectory, as compared to no high school diploma (aOR 1.31, 95\% 1.05-1.62; OR 1.35, 95\% CI 1.15-1.60; OR 1.75, 95\% CI 1.37-2.22 for high school diploma, college/CEGEP, and university degree, respectively). Being an immigrant was associated with higher odds of membership in this trajectory (aOR 1.46, 95\% CI 1.11-1.93). Living outside of
Comorbidities (continuous scale)

Place of diagnosis (ref: hospital)

Sex (ref: female)

Age group (ref: 20-40)

Education (ref: < high school)

Immigrant (ref: not an immigrant)

Region (ref: Montreal)

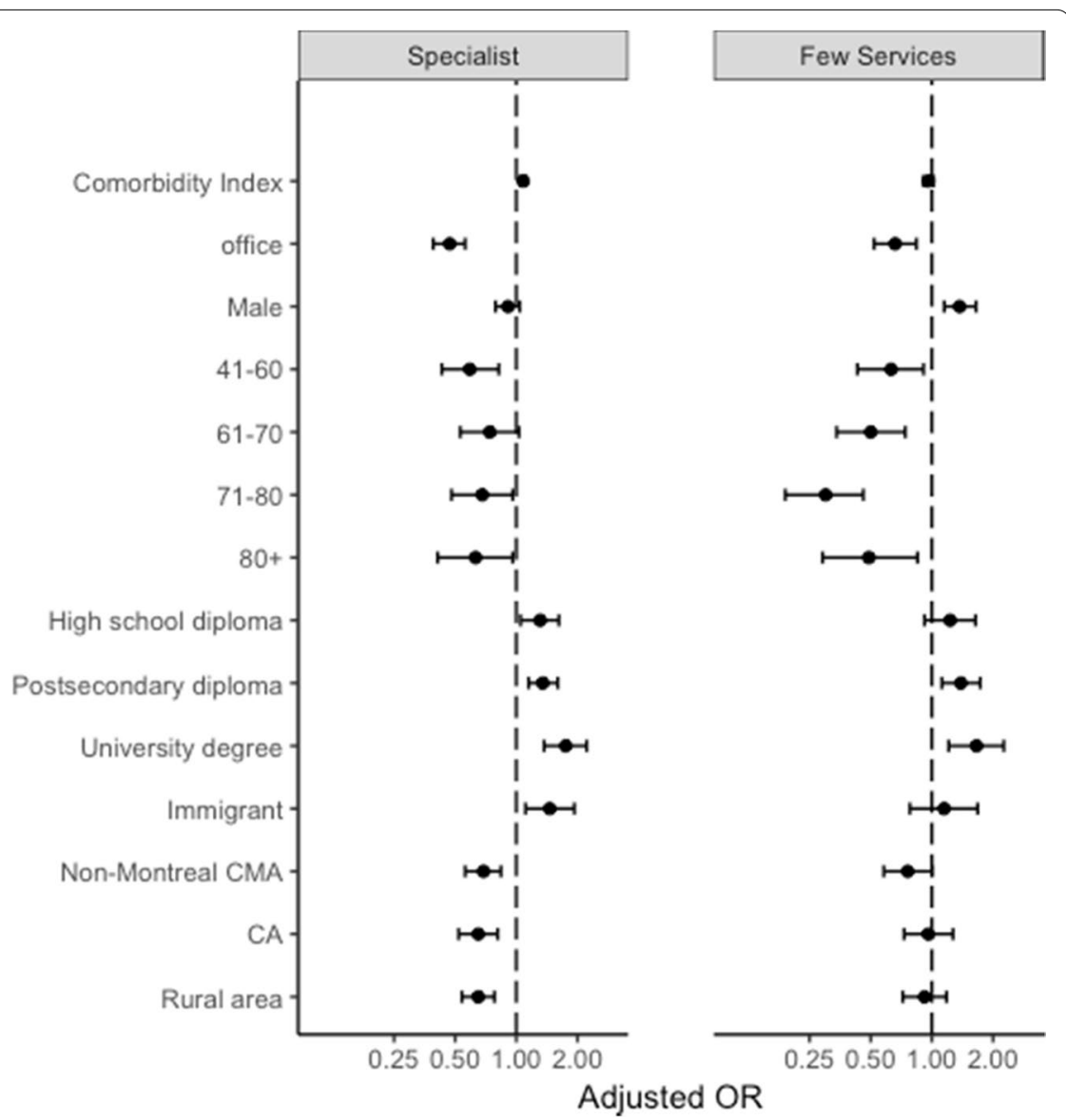

Fig. 3 Multinomial logistic regression model of factors associated with trajectory group membership, in reference to the Regular Family Physician Trajectory. $C M A=$ Census Metropolitan Area; $C A=$ Census Agglomeration 
the Montreal urban area was associated with lower odds of following this Specialist Trajectory (aOR 0.69, 95\% CI 0.56-0.84; OR 0.65, 95\% CI 0.52-0.81; OR 0.65, 95\% CI 0.54-0.78 for non-Montreal CMA, CA, and rural area, respectively).

\section{Few services vs. family physician}

Being diagnosed in an office as compared to in hospital was associated with lower odds of following the Few Services Trajectory, compared to the Regular FP Trajectory (aOR 0.66, 95\% CI 0.52-0.84). Males had higher odds of membership in the Few Services Trajectory than females (aOR 1.37, 95\% CI 1.15-1.65). Increasing age groups had lower odds of membership in this trajectory (compared to ages 20-40: aOR 0.63, 95\% CI 0.43-0.91 for ages 41-60; aOR 0.50, 95\% CI $0.34-0.74$ for ages $61-70$; aOR 0.30 , 95\% CI $0.19-0.46$ for ages 71-80; and aOR $0.49,95 \%$ CI $0.29-0.85$ for ages $80+$ ). Having a college/ CEGEP or university degree, compared to no high school diploma, was positively associated with membership in the Few Services Trajectory (aOR 1.39, 95\% CI 1.12-1.73; OR 1.66, 95\% CI 1.21-2.26 respectively). While living in an urban area other than Montreal was associated with lower odds of membership in the Few Services Trajectory, this was borderline non-significant (aOR 0.76, 95\% CI 0.58-1.00). Other areas of residence showed no differences in the few services membership pattern compared to residence in Montreal.

\section{Discussion}

Our study aimed to identify a typology of care trajectories following diabetes diagnosis and to explore sociodemographic variables associated with trajectory-type membership. From this analysis, we describe three distinct patterns of all-cause health care utilization in the first 2 years following a diagnosis of diabetes in a cohort of people in Quebec. Forty-three percent of our sample followed a trajectory defined predominantly by regular FP care. Another $41 \%$ followed a trajectory comprising mostly specialist care with some involvement of regular FPs as well as "new" (non-regular) FPs. The rest (16\%) followed a pattern characterized by few services overall. Those with few services spent an approximately equivalent amount of time in the hospital and emergency care state as those individuals following the Regular FP Trajectory.

Various methods have previously been used to explore and describe different patterns in healthcare utilization. One recent study measured care seeking as irregular provider contact, regular specialized care, and regular generalized care for diabetes based on yearly care patterns [23]. Using group-based trajectory modeling over an 11-year period, they found seven trajectories (persistent irregular use, generalized to irregular, irregular to generalized, persistent generalized, generalized to specialized, specialized to generalized, and persistent specialized); membership to which differed by age, SES, and residential location [23]. Another approach applied a tailored state sequence analysis to study where a service was undertaken, by which specialist, and for which diagnosis among a cohort of patients with chronic obstructive pulmonary disease followed for 1 year [24]. Five trajectory types, corresponding to low healthcare utilization, moderate healthcare utilization, and three high utilization groups with predominantly respiratory diagnoses, cardiovascular diagnoses, and other diagnoses, respectively [24]. Our analysis took a slightly different approach in assigning care states a lasting duration. Nevertheless, our results indicate a low utilization group, a moderate utilization group dominated by regular family physician care, and a higher utilization group dominated by specialist care.

Our results suggest a path dependence in access to care. Individuals in the Specialist Trajectory spent nearly twice as long overall in the hospital and emergency care state. It is conceivable that patients connect with specialists during a hospital encounter, and then potentially bypass the family physician or otherwise have direct follow ups with specialists [25]. Indeed, diagnosis in a physician's office (i.e., outside of hospital) was associated with lower odds of following the Specialist Trajectory than diagnosis in hospital, as compared to the Regular FP Trajectory. This may reflect differences in the pre-diagnosis trajectory, suggested also by the fact that comorbidities were statistically significantly higher in the Specialist Trajectory than the Regular FP Trajectory. People with the Few Services Trajectory showed no significant differences in comorbidities compared to those with the Regular FP Trajectory.

The second part of our objective was to investigate inequalities in care trajectories. We have argued that a trajectory favouring continuity of care with a regular FP is optimal, as it most closely represents the recommended chronic care management model of PCSCs [4]. While we did observe some socially patterned differences in the odds of following different types of trajectories, we did not find strong evidence of social status privileging access to the Regular FP Trajectory. Immigrants and people with higher education had higher odds of following the Specialist Trajectory as compared to the Regular FP Trajectory. People with higher education also had higher odds of following the Few Services trajectory, which may seem a counterintuitive finding. This result could also be reflective of other dynamics including less frequent visits to a FP due to higher participation in diabetes management programs run outside the family physician's clinic, or a higher perceived sense of control over condition 
management recommendations. However, a recent Canadian study has found that people with higher education reported greater difficulties in accessing health care, suggesting that there may be further explanations to explore. After accounting for comorbidities, older people had lower odds of following the Specialist Trajectory, as well as lower odds of following the Few Services Trajectory, likely because they already had access to a family physician.

Men were more likely than women to follow the Few Services Trajectory, as compared to the Regular FP Trajectory, a finding that is supported by studies showing that men are less likely to visit an FP compared to women [26].

Previous studies have shown a higher rate of specialist visits with higher income and education levels in Canada, even after adjusting for health care need, which suggests the potential influence of these factors on the likelihood of a referral from primary care [27]. In the present study, we found that residents of areas outside of the large urban centre of Montreal were also less likely to follow the Specialist Trajectory. This could be attributable to geographic disparities in access to a family physician the proportion of people in Montreal with a regular family physician is lower than elsewhere in the province [28] - or to a supply effect of the higher concentration of specialists in the urban centre of Montreal [29].

In our analysis, immigrants were more likely to follow the Specialist Trajectory than non-immigrants, as compared to the Regular FP Trajectory. Immigrants may be less likely to have access to a regular family physician [30], although other studies have not demonstrated this difference [31]. Of note, we found that immigrants were no more likely to follow the Few Services Trajectory than non-immigrants after adjusting for place of residence. This suggests that the concentration of immigrants in a large urban area (Montreal, QC) was not driving the effect. However, grouping all immigrants into one category undoubtedly glosses over nuances.

\section{Strengths and limitations}

This study uses a novel cohort in Quebec of survey participants linked to administrative data, which allows richer socio-demographic data than is normally available in administrative data only.

This analysis was not designed to test causal differences in following different trajectory types. However, the socio demographic factors we explored here are all relatively time invariant and would be determined prior to the start of the trajectory. We have not yet assessed outcomes associated with trajectory types membership, which limits our ability to draw conclusions about their effectiveness. This will be the basis of our subsequent work in this area.

We used education instead of income as a proxy measure of SES; as the CCHS interview could occur at varying intervals around the start of the trajectory, we did not have an income measure consistent with the start of the trajectory. We thus opted for measures that would be fixed in time. In support of this decision, we note that in a universal care system with a single government payer, income would be less expected to impact access to or utilization of care. Education level, on the other hand, may influence health literacy and patients' abilities to navigate the health system. Indeed, previous studies have found education to show stronger associations with health service utilization than income [32].

We defined a regular FP as the one providing at least half of an individual's family medicine visits in the past year. While stricter definitions have been used [33], we still see important differences emerge with our broader approach. Importantly though, we are lacking information on registration with a groupe de médecin de famille (family medicine group, GMF), so we cannot rule out that the "non-regular" FP visits are not with another physician in an individual's GMF. GMFs were designed to improve continuity of care, even when an individual is not able to see their own regular physician.

Finally, we could not distinguish between type 1 and 2 diabetes with our algorithm; however, given our age criteria of individuals 20 years old and above it is reasonable to assume that our cohort is primarily composed of individuals with type 2 . Type 1 diabetes accounts for $5-10 \%$ of all diabetes diagnoses, and occurs mostly in children [34]. Our approach to the analysis assessed all-cause health care utilization. It will be important in subsequent work to identify the intensity of diabetes-specific utilization within and among trajectory groups, and to distinguish this from other primary diagnoses associated with healthcare encounters.

Our approach to measure healthcare use maintains daily granularity. Alternatives include measuring use in wider time blocks, such as monthly. However, this would necessitate defining a hierarchy to account for the potential of multiple care states during the time period. As we were equally interested in the presence of each state, we wanted to avoid making hierarchical determinations. However, this daily approach may have been too granular to allow for changing longitudinal trends in care states to be detected (such as shifts from specialist to family physician care, or from new to regular family physician care, over time).

The benefit of sequence analysis is the ability to simultaneously consider the evolution of care interactions over time, without focusing explicitly on transitions between 
states - the trajectories are analysed as a static object, while allowing for dynamic patterns within [35]. This is a significant benefit in chronic condition health care utilization research, where transitions between care states may be of less interest than the overall pattern of care.

\section{Potential policy implications}

The medical home model of health care prioritizes strong connections to a primary care provider or team, with support of specialist consultants as necessary. The Regular FP care trajectory that we used as reference fits well within this model and with that of PCSCs, which stipulates that most treatment, monitoring, and support for diabetes can and should occur in the primary care setting $[17,36]$. To note, this care trajectory is not only deemed optimal for the patient, but also for the system, as it prevents unnecessary, more costly, and potentially invasive care [4].

Our analysis identified a pattern of specialist-predominant services that would generally not conform to the PCSC model. In fact, specialist care is not uniformly associated with better outcomes following a diabetes diagnosis, despite being associated with the use of appropriate diabetes-specific treatments [37]. Efforts could be encouraged to link patients diagnosed in hospital with suitable family physicians willing to take charge of the chronic issue management, if a family physician is not already assigned. In Quebec, this could be implemented through the guichet d'accès à un médecin de famille (GAMF), which is a centralized intake and waitlist system for individuals seeking a family physician [38].

\section{Conclusion}

This analysis of a survey-based cohort linking to health administrative records found three relatively stable trajectory types to be prevalent among adults with a new diagnosis of diabetes. Further understanding how sociodemographic and other factors relate to a patient's experience with following typical trajectories of care will serve to identify ways to enhance the patient's experience, the quality and appropriateness of care, and ultimately reinforce the ability to steer patients towards care trajectories best aligned with the PCSC care approach.

\section{Supplementary Information}

The online version contains supplementary material available at https://doi. org/10.1186/s12913-021-07450-9.

Additional file 1.

\section{Acknowledgements}

The authors thank The Quebec Population Health Research Network (QPHRN) and the Cardiometabolic Health, Obesity and Diabetes Research Network (CMDO) for their contribution to the financing of this publication.
AQV and AL are members of the TORSADE cohort working group. The full list of members is: Alain Vanasse (leader), Gillian Bartlett, Lucie Blais, David Buckeridge, Manon Choinière, Catherine Hudon, Anaïs Lacasse, Benoit Lamarche, Alexandre Lebel, Amélie Quesnel-Vallée, Pasquale Roberge, Valérie Émond, Marie-Pascale Pomey, Mike Benigeri, Anne-Marie Cloutier, Marc Dorais, Josiane Courteau, Mireille Courteau, Stéphanie Plante, Pierre Cambon, Annie Giguère, Isabelle Leroux, Danielle St-Laurent, Denis Roy, Jaime Borja, André Néron, Geneviève Landry, Jean-François Ethier, Roxanne Dault, Marc-Antoine CôtéMarcil, Pier Tremblay, Sonia Quirion.

\section{Authors' contributions}

AQV and AL conceived the project, based on the Torsade Cohort umbrella study. RM and AQV designed the analysis. RM conducted the analysis and wrote the manuscript. AQV added insight to the interpretation and critical revision of the manuscript. LL made important contributions to the study design, data analysis, and the manuscript. AL critically revised the manuscript. All authors take responsibility for the integrity of the work. The authors read and approved the final manuscript.

\section{Funding}

This work was also supported by grants provided by Canadian Institutes of Health Research (Funding reference numbers 162482 and 156043).

\section{Availability of data and materials}

The data that support the findings of this study are available from the Institut de la statistique du Québec but restrictions apply to the availability of these data, which were used under license for the current study, and so are not publicly available. Data are however available from the authors upon reasonable request and with permission of $\mathrm{ISQ}$, authorization from the $\mathrm{CAl}$, and other appropriate approvals from relevant data stewards.

\section{Declarations}

Ethics approval and consent to participate

This study was approved by the Research Ethics Board of McGill University, and by the Commission d'accès à l'information of Québec.

\section{Consent for publication}

Not applicable.

\section{Competing interests}

The authors declare that they have no conflicts of interest.

\section{Author details}

${ }^{1}$ McGill Observatory on Health and Social Services Reforms, Montreal, Canada. ${ }^{2}$ Department of Epidemiology, Biostatistics, and Occupational Health, McGill University, Montreal, Canada. ${ }^{3}$ Centre for Research on Planning and Development (CRAD), Laval University, Quebec G1V 0A6, Canada. ${ }^{4}$ Evaluation Platform on Obesity Prevention, Quebec Heart and Lung Institute Research Center, Quebec G1V 4G5, Canada. ${ }^{5}$ Department of Sociology, McGill University, Montreal, Canada.

Received: 27 October 2021 Accepted: 22 December 2021

Published online: 31 January 2022

References

1. World Health Organization. Diabetes Fact Sheet [Internet]. 2020 [cited 2020 Jun 24]. Available from: https://www.who.int/news-room/factsheets/detail/diabetes

2. Vanasse A, Courteau M, Ethier J-F. The ' 6 W' multidimensional model of care trajectories for patients with chronic ambulatory care sensitive conditions and hospital readmissions. Public Health. 2018;157:53-61.

3. Laiteerapong N, Karter AJ, Moffet HH, Cooper JM, Gibbons RD, Liu JY, et al. Ten-year hemoglobin A1c trajectories and outcomes in type 2 diabetes mellitus: the Diabetes \& Aging Study. J Diabetes Complicat. 2017;31(1):94-100. 
4. Gibbons DC, Bindman AB, Soljak MA, Millett C, Majeed A. Defining primary care sensitive conditions: a necessity for effective primary care delivery? J R Soc Med. 2012;105(10):422-8.

5. College of Family Physicians of Canada. A new vision for Canada: Family Practice - The Patient's Medical Home 2019 [Internet]. Mississauga: College of Family Physicians of Canada; 2019. [cited 2020 Nov 1]. Available from: https://patientsmedicalhome.ca/files/uploads/PMH_VISION2019_ ENG_WEB_2.pdf

6. Cho KH, Nam CM, Choi Y, Choi J-W, Lee S-H, Park E-C. Impact of continuity of care on preventable hospitalization of patients with type 2 diabetes: a nationwide Korean cohort study, 2002-10. Int J Qual Health Care. 2016;28(4):478-85.

7. Brown AF, Ettner SL, Piette J, Weinberger M, Gregg E, Shapiro MF, et al. Socioeconomic position and health among persons with diabetes mellitus: a conceptual framework and review of the literature. Epidemiol Rev. 2004;26(1):63-77.

8. Agardh E, Allebeck P, Hallqvist J, Moradi T, Sidorchuk A. Type 2 diabetes incidence and socio-economic position: a systematic review and metaanalysis. Int J Epidemiol. 2011;40:804-18.

9. Rossi MC, Cristofaro MR, Gentile S, Lucisano G, Manicardi V, Mulas MF, et al. Sex disparities in the quality of diabetes care: biological and cultural factors may play a different role for different outcomes: a cross-sectional observational study from the AMD annals initiative. Diabetes Care. 2013;36(10):3162-8.

10. Lovshin JA, Shah BR. Inadequate screening for retinopathy among recent immigrants with type 2 diabetes despite universal health care: a population-based study. J Diabetes Complicat. 2017;31(4):664-8.

11. Vanasse A, Chiu YM, Courteau J, Dorais M, Bartlett G, Zawaly K, et al. Cohort profile: the care trajectories—enriched data (TorSaDE) cohort. Int J Epidemiol. 2020;00(00):9.

12. Tarasuk V, Cheng J, de Oliveira C, Dachner N, Gundersen C, Kurdyak P. Association between household food insecurity and annual health care costs. CMAJ. 2015;187(14):E429-36.

13. Di Giulio P, Impicciatore R, Sironi M. The changing pattern of cohabitation: a sequence analysis approach. Demogr Res. 2019;40:1211-48.

14. Chen G, Khan N, Walker R, Quan H. Validating ICD coding algorithms for diabetes mellitus from administrative data. Diabetes Res Clin Pract. 2010:89(2):189-95.

15. Asghari S, Courteau J, Carpentier AC, Vanasse A. Optimal strategy to identify incidence of diagnostic of diabetes using administrative data. BMC Med Res Methodol. 2009;28(9):62.

16. Nakhla M, Bell LE, Wafa S, Dasgupta K. Improving the transition from pediatric to adult diabetes care: the pediatric care provider's perspective in Quebec, Canada. BMJ Open Diabetes Res Care. 2017;5(1):e000390.

17. Diabetes Canada. Clinical Practice Guidelines, vol. 42; 2018. p. 342.

18. Simard M, Sirois C, Candas B. Validation of the combined comorbidity index of Charlson and Elixhauser to predict 30-day mortality across ICD-9 and ICD-10. Med Care. 2018;56(5):441-7.

19. Schneeweiss S, Wang PS, Avorn J, Glynn RJ. Improved comorbidity adjustment for predicting mortality in Medicare populations. Health Serv Res. 2003;38(4):1103-20.

20. Gabadinho A, Ritschard G, Müller NS, Studer M. Analyzing and visualizing state sequences in R with TraMineR. J Stat Softw. 2011;40(4) [cited 2018 Jun 7]. Available from: http://www.jstatsoft.org/v40/i04/.

21. Lesnard L. Optimal Matching and Social Sciences. Halshs-00008122. 2006;27.

22. Aisenbrey S, Fasang AE. New life for old ideas: the "second wave" of sequence analysis bringing the "course" Back into the life course. Sociol Methods Res. 2010;38(3):420-62.

23. Tsai T, Huang N, Lin I-F, Chou Y-J. Variation in the 11-year trajectories of medical care seeking behaviors in diabetes patients under a single payer system: persisting gaps to be filled. BMC Health Serv Res. 2019;19(1):1-11.

24. Vanasse A, Courteau J, Courteau M, Benigeri M, Chiu YM, Dufour I, et al. Healthcare utilization after a first hospitalization for COPD: a new approach of state sequence analysis based on the " $6 \mathrm{~W}$ " multidimensional model of care trajectories. BMC Health Serv Res. 2020;20(1):177.

25. Glazier RH, Agha MM, Moineddin R, Sibley LM. Universal health insurance and equity in primary care and specialist office visits: a population-based study. Ann Fam Med. 2009;7(5):396-405.
26. Wang Y, Hunt K, Nazareth I, Freemantle N, Petersen I. Do men consult less than women? An analysis of routinely collected UK general practice data. BMJ Open. 2013;3(8):e003320.

27. Dunlop S, Coyte PC, Mclsaac W. Socio-economic status and the utilisation of physicians'services: results from the Canadian National Population Health Survey. Soc Sci Med. 2000;51(1):123-33.

28. Ministère de la santé et services sociaux Québec. Accès aux services médicaux de première ligne - Professionnels de la santé [Internet]. 2019 [cited 2020 Nov 2]. Available from: https://www.msss.gouv.qc.ca/profe ssionnels/statistiques-donnees-services-sante-services-sociaux/accespremiere-ligne/.

29. Wennberg JE. Unwarranted variations in healthcare delivery: implications for academic medical centres. BMJ. 2002;325(7370):961-4.

30. Difficulty accessing health care services in Canada [Internet]. [cited 2019 Oct 31]. Available from: https://www150.statcan.gc.ca/n1/pub/82-624-x/ 2016001/article/14683-eng.htm.

31. Quesnel-Vallée A, Setia MS, Abrahamowicz M, Tousignant P, Lynch J. Access to health Care in Canadian Immigrants: a longitudinal study of the National Population Health Survey. Health Soc Care Community. 2011;19(1):70-9.

32. Sorts $\varnothing$ C, Lauridsen J, Emneus M, Green A, Jensen PB. Socioeconomic inequality of diabetes patients' health care utilization in Denmark. Health Econ Rev. 2017;7(1):21.

33. Provost S, Pérez J, Simard B, Équipe santé des populations et services de santé (Québec), Agence de la santé et des services sociaux de Montréal, Secteur services préventifs en milieu clinique, et al. Affiliation à un médecin de famille: une mesure à partir des banques de données médico-administratives [Internet]. Montréal: Équipe santé des populations et services de santé, Secteur services préventifs en milieu clinique, Direction de santé publique de l'Agence de la santé et des services sociaux de Montréal et Direction de l'analyse et de l'évaluation des systèmes de soins et services, Institut national de santé publique du Québec; 2013. [cited 2020 Aug 12]. Available from: http://collections.banq.qc.ca/ark:/ $52327 / 2317079$

34. Public Health Agency of Canada. Diabetes in Canada: facts and figures from a public health perspective. Ottawa: Public Health Agency of Canada; 2011. p. 112

35. Ritschard G, Studer M. Sequence Analysis: Where Are We, Where Are We Going? In: Sequence Analysis and Related Approaches Life Course Research and Social Policies [Internet]. Cham: Springer; 2018. Available from: https://doi.org/10.1007/978-3-319-95420-2_1.

36. Bojadzievski T, Gabbay RA. Patient-centered medical home and diabetes. Diabetes Care. 2011;34(4):1047-53.

37. McAlister FA, Majumdar SR, Eurich DT, Johnson JA. The effect of specialist care within the first year on subsequent outcomes in 24232 adults with new-onset diabetes mellitus: population-based cohort study. Qual Saf Health Care. 2007;16(1):6-11.

38. Breton M, Brousselle A, Boivin A, Loignon C, Touati N, Dubois C-A, et al. Evaluation of the implementation of centralized waiting lists for patients without a family physician and their effects across the province of Quebec. Implement Sci. 2014:9(1):117.

\section{Publisher's Note}

Springer Nature remains neutral with regard to jurisdictional claims in published maps and institutional affiliations. 